\title{
Time- and space-resolved optical Stark spectroscopy in the afterglow of laser-produced tin-droplet plasma
}

\author{
J. Scheers $\odot,{ }^{1,2}$ R. Schupp ${ }^{\oplus},{ }^{1}$ R. Meijer $\odot,{ }^{1,2}$ W. Ubachs $\odot{ }^{1,2}$ R. Hoekstra ${ }^{\circledR},{ }^{1,3}$ and O. O. Versolato ${ }^{1}{ }^{1,2, *}$ \\ ${ }^{1}$ Advanced Research Center for Nanolithography, Science Park 110, 1098 XG Amsterdam, The Netherlands \\ ${ }^{2}$ Department of Physics and Astronomy, and LaserLaB, Vrije Universiteit, De Boelelaan 1081, 1081 HV Amsterdam, The Netherlands \\ ${ }^{3}$ Zernike Institute for Advanced Materials, University of Groningen, Nijenborgh 4, 9747 AG Groningen, The Netherlands
}

(Received 2 May 2020; accepted 15 June 2020; published 9 July 2020)

\begin{abstract}
The afterglow emission from Nd:YAG-laser-produced microdroplet-tin plasma is investigated, with a focus on analyzing Stark effect phenomena and the dynamical evolution of the plasma. Time- and space-resolved optical imaging spectroscopy is performed on 11 lines from Sn I-IV ions, in the 315-425-nm wavelength range. Stark shift-to-width ratios serve as the basis for unambiguous experimental tests of atomic physics theory predictions. Experiment and theory, where available, are found to be in poor agreement, and are in disagreement regarding the sign of the ratio in several cases. Spectroscopic measurements of the Stark widths in tandem with SahaBoltzmann fits to Sn I and Sn II lines, establish the evolution of the local temperature and density of the plasma afterglow, 20-40 ns after the end of the 15-ns-long temporally box-shaped laser pulse. A clear cool-down from $\sim 2$ to $1 \mathrm{eV}$ is observed of the plasma in this time window, having started at $\sim 30 \mathrm{eV}$ when emitting extremeultraviolet (EUV) light. An exponential reduction of the density of the plasma from $\sim 10^{18}-10^{17} e^{-} \mathrm{cm}^{-3}$ is observed in this same time window. Our work is relevant for understanding the dynamics of the decaying, expanding plasma in state-of-the-art EUV nanolithography machines.
\end{abstract}

DOI: 10.1103/PhysRevE.102.013204

\section{INTRODUCTION}

Laser-produced tin plasma is used in state-of-the-art extreme-ultraviolet (EUV) nanolithography machines where highly charged tin ions produce the required EUV light. Hot $(10-100 \mathrm{eV})$ and dense $\left(10^{19}-10^{21} e^{-} \mathrm{cm}^{-3}\right)$ plasma is produced when molten Sn microdroplets are illuminated by high-energy laser pulses [1]. The responsible ions for emitting EUV photons near $13.5 \mathrm{~nm}$ are $\mathrm{Sn} \mathrm{XI-Sn} \mathrm{XV}$ with their resonance transitions $4 p^{6} 4 d^{m}-4 p^{5} 4 d^{m+1}$ and $4 d^{m}-4 d^{m-1} 4 f(m=$ 4-0) [1-5]. Besides the lithographically applicable EUV radiation, the laser-matter interaction produces a violently expanding plasma with a broad range of charge states down to neutral Sn I.

Besides the measurement of kinetic energies of ions penetrating into the far field [6], optical spectroscopy is a convenient and versatile plasma diagnostic tool for understanding and monitoring the plasma in its expansion phase, where it has lower temperature and density. Line emission from the relatively low charge states Sn I-IV can readily be identified in the optical domain. Intensities, shapes, and shifts of the lines can provide information about the local plasma conditions, such as temperature and electron density.

Light emission from lowly charged tin ions is found in a wide variety of plasma, ranging from the here relevant

\footnotetext{
*versolato@arcnl.nl
}

Published by the American Physical Society under the terms of the Creative Commons Attribution 4.0 International license. Further distribution of this work must maintain attribution to the author(s) and the published article's title, journal citation, and DOI. laser-produced Sn plasmas for generating EUV light, to potential plasma heat shields for fusion reactors [7-10], and astrophysical plasma [11-22]. Spectral lines of $\mathrm{Sn}$ in the optical range are found in various collections by the Atomic Spectra Database maintained by the National Institute of Standards and Technology [23]. Additional, extended lists of line positions are available for Sn II [24], Sn III [25], and Sn IV [26].

The Stark effect, the broadening and shifting of lines mostly due to the interaction of the tin ions with plasma electrons at the densities and temperatures relevant for this work, can be employed to gauge the plasma density using characteristic features of the plasma line emission [27-33]. Methods based on the Stark effect require knowledge of the local plasma temperature as well as input on the Stark shift and width parameters (see, e.g., Refs. [34,35]), typically stemming from theory. Stark broadening parameters for Sn can be found in various compilations [36-39]. Detailed spectroscopic work and theoretical calculations on Stark broadening are available for Sn I [40,41], Sn II [42-47], Sn III [48,49], Sn I and II [50-54], Sn II and III [29], Sn II, III, IV [55], and Sn IV [56-59]. Relevant and typically studied electron temperatures are between 1 and $5 \mathrm{eV}$. Less information is available on Stark shifts. Calculation of the shift parameter is difficult due to possible cancellations of terms in the formulas [34,60]. All in all, a large body of literature is available, enabling extraction of the physical conditions of plasma expansion. It provides the basic scaffolding for our studies of the afterglow emission from Nd:YAG-laser-produced microdroplet-tin plasma.

Specifically, we present time- and space-resolved spectroscopy of 11 lines, in a 315-425-nm wavelength range, of Sn I-IV ions residing in the expanding plasma, several tens of nanoseconds (in the afterglow) after the end of the 15-ns-long, 
temporally box-shaped laser pulse. The measured Stark shifts and widths enable obtaining the shift-over-width ratios, which are largely independent of temperature and density at the here relevant densities and $\sim 1-e V$-level temperatures in this afterglow phase. These ratios serve as the basis for unambiguous experimental tests of the available theoretical predictions. Next, we use the literature data for the Stark width parameters for eight lines of Sn I-IV ions in combination with our timeand space-resolved spectroscopic observations to diagnose the time evolution of the density and temperature of the plasma in this afterglow phase.

\section{EXPERIMENT}

The experimental setup consists of a vacuum vessel equipped with multiple viewports for diagnostics purposes. A droplet generator, attached to the top of the setup, dispenses molten tin droplets with a constant diameter of $46 \mu \mathrm{m}$. Droplets, ejected at a repetition rate of $10 \mathrm{kHz}$, fall through a laser sheet created by a HeNe laser. Scattered laser light, observed on a photon multiplier tube, triggers the plasma illumination laser and acquisition systems. The tin droplets are irradiated with laser pulses from an in-house built Nd:YAG laser system [61]. The laser is operated at a wavelength of $1064 \mathrm{~nm}$ and is capable of generating arbitrary pulse shapes. In the experiments, temporally box-shaped pulses of $15 \mathrm{~ns}$ duration with a flat-top spatial beam profile (115 $\mu \mathrm{m}$ in diameter) and energies of $270 \mathrm{~mJ} /$ pulse are used at a $10-\mathrm{Hz}$ repetition rate. The resulting laser intensity of $1.7 \times 10^{11} \mathrm{~W} / \mathrm{cm}^{2}$ is relevant for the efficient production of EUV light [62]. Hydrogen is introduced in the vacuum vessel as a buffer gas, at a pressure of about 1 mbar, to prevent contamination of viewports. The presence of the buffer gas did not have any measurable influence on the optical spectral emission characteristics as compared to vacuum conditions on the time- and spatial-length scales studied in this work. More detailed descriptions of the experimental setup can be found in Refs. [62,63].

Imaging spectroscopy is performed by analyzing light emitted from the generated plasma, observed perpendicularly to the laser propagation direction and at $30^{\circ}$ with respect to the horizontal plane. The plasma light first traverses through a quartz vacuum viewport after which it is collected by a biconvex lens with a focal length of $100 \mathrm{~mm}$. This lens is placed outside of the vacuum at a distance that enables creating a one-to-one projection image of the plasma onto a linear fiber array, with an imaging resolution of several tens of micrometers and an approximate depth-of-field of $\sim 1 \mathrm{~mm}$ at a representative $350-\mathrm{nm}$ wavelength. The fiber array contains 35 quartz fibers with a $100-\mu \mathrm{m}$ core diameter, in an ordered linear-to-linear configuration. Cladding around each fiber core adds up to an effective diameter of $125 \mu \mathrm{m}$, leading to a total size of the fiber array of $4.4 \mathrm{~mm}$. The fiber size sets the effective field of view of the optical system and the spatial resolution is set by the $100-\mu \mathrm{m}$ core diameter. The imaging system is realigned for each grating position to correct for chromatic aberrations introduced by the lens, with a position accuracy of approximately \pm 1 fiber core, or $\pm 125 \mu \mathrm{m}$. The fiber array is attached to an imaging spectrometer (Princeton
Instruments SCT320) using a reimaging adapter that images the fiber array onto the slit of the spectrometer.

A grating with a line spacing of 1200 lines per millimeter, optimized for emission in the ultraviolet range, is employed. A total spectral range from 250 to $430 \mathrm{~nm}$ is covered, with a window of about $30 \mathrm{~nm}$ for a single frame. We focus our studies on the 315-425-nm wavelength range where bright Sn lines are observed, and well-resolved Sn lines (also when significantly Stark broadened) are observed. For wavelengths longer than $400 \mathrm{~nm}$ a long-pass filter is used to block secondorder diffraction from the grating. Wavelength calibration is performed using known $\mathrm{Hg}$ lines after exposure to a calibration light source, as well as known "in vivo" Sn lines. The instrumental, Gaussian linewidth is determined from the $\mathrm{Hg}$ lines and ranges from 0.17 at $250 \mathrm{~nm}$ to $0.14 \mathrm{~nm}$ at $430 \mathrm{~nm}$, at full width half maximum (FWHM). The calibration uncertainty of absolute line positions is $0.02 \mathrm{~nm}$, dominated by limitations set by the sparsity of available $\mathrm{Hg}$ lines, and by the complexity of using modestly broadened and in some cases overlapping Sn lines for calibration purposes. Furthermore, imaging artifacts such as finite tilts can influence wavelength calibration but these are below the $0.02 \mathrm{~nm}$ level.

The resulting image on the camera mounted to the spectrometer thus contains spectral information as dispersed by the grating in the spectrometer along the horizontal axis and the image of the fiber cores along the vertical axis. For a time-resolved spectral detection an intensified CCD camera (4Picos ICCD) is used. The temporal evolution of the transient plasma is captured by setting the width of a box-gated window at $10 \mathrm{~ns}$. Plasma light is integrated for 1000 laser shots before readout in order to capture an adequate amount of light. The use of the ICCD's multichannel plate guarantees full suppression of light produced outside of the 10-ns gated time. Use of the time-resolved CCD camera thus leads to three-dimensional information on the plasma: space, time, and wavelength.

In Fig. 1 the projection geometry is schematically depicted. In addition, the figure shows a two-dimensional image of space-resolved spectra of two adjacent Sn lines taken at $t=$ $50 \mathrm{~ns}$ after the start of the laser pulse, extending over seven cores, therewith spanning a spatial image of the plasma of about $0.9 \mathrm{~mm}$. The time evolution of this part of the spectrum is depicted also.

\section{MEASUREMENTS}

In the following, the Sn I-IV charge-state evolution over time, as obtained from the time-resolved measurements, is discussed first. Next, the Stark broadening and shifts of individual and isolated lines of the four charge states are studied and used to obtain shift-to-width ratios. The results are compared to theory predictions where available. Subsequently, in Sec. IV, experimental results pertaining to Stark widths of lines from Sn I-IV are used in tandem with Saha-Boltzmann fits to line strengths, to obtain time-resolved temperature and density evolution of the plasma.

\section{A. Charge-state evolution}

Time-resolved spectral measurements are presented in Fig. 2. Spectra of plasma light captured by fiber core $\mathbf{c}$ are 

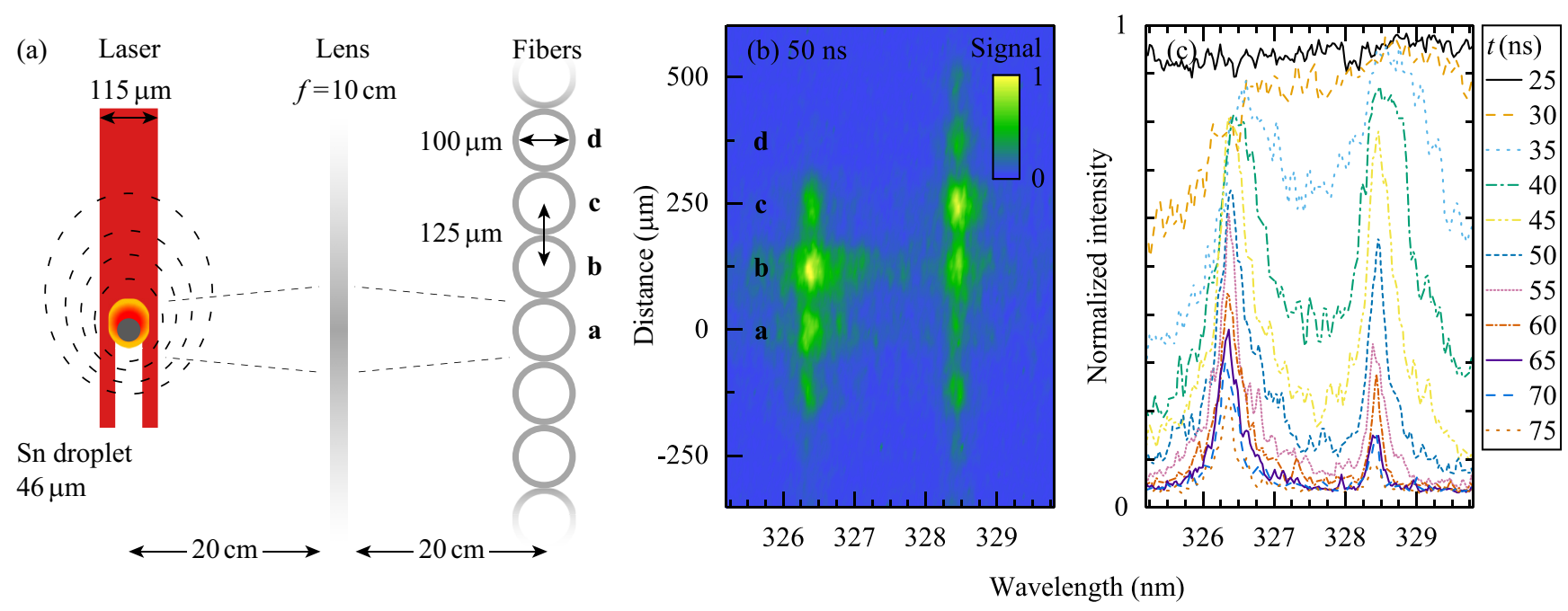

FIG. 1. Schematic layout of the experimental setup. Panel (a): Geometry of the optical system to project plasma emission onto the fiber array. The here relevant fiber cores are labeled a-d. Panel (b): ICCD image capturing emission of Sn I (at $\lambda=326 \mathrm{~nm}$ ) and Sn II (at $\lambda=328 \mathrm{~nm}$ ) lines from seven fiber cores, covering an approximately $0.9 \mathrm{~mm}$ spatial extent of the plasma as a function of wavelength, at time $t=50 \mathrm{~ns}$. Transitions are detailed in Table I. Panel (c): Time-resolved spectra from fiber core b, covering the temporal evolution from 25 to 75 ns.

shown (see Fig. 1). Lines belonging to Sn I-IV are depicted separately to highlight the evolution of the charge-state distribution with time. The time of the first measurement frame in which plasma radiation is observed defines $t=0$, with a systematic uncertainty of approximately $\pm 5 \mathrm{~ns}$. During the 15-ns-long laser pulse, and in a similarly long period thereafter, strong continuum emission is observed in fiber cores $\mathbf{a}$ and $\mathbf{b}$. Continuum emission is typically first observed in fiber core a and soon after it also is strongly visible in fiber core b. After this period line emission emerges and starts to dominate the spectrum. Strongly broadened lines belonging to
Sn III and IV are first observed at $t=25 \mathrm{~ns}$. It takes about $10 \mathrm{~ns}$ longer before individual Sn I and II lines can be identified. The spectral width and intensity of all lines quickly reduces with time. Besides the strong broadening, the high plasma density is also apparent through the self-absorption feature observed in a broadened Sn III line at the earliest time (see Fig. 2). We note that the large depth of field of our experiment effectively entails an integration of the emission over a plasma column spanning a large density and temperature range. However, most of the emission can be expected to originate from the densest part of the plasma, singling out the dense plasma

TABLE I. Stark width and shift parameters for selected lines in Sn I-IV. The Stark widths (at FWHM) are given at an electron density of $10^{17} e^{-} \mathrm{cm}^{-3}$ and are scaled to a temperature of $1 \mathrm{eV}$ (given a $T^{-1 / 2}$ dependence). Shift-over-width ratios $d / w$ are the resulting slopes of fitted linear functions on concatenated data, shown for selected lines in Fig. 3. The uncertainty on the fitted slope is listed between brackets. A positive shift is towards longer wavelength.

\begin{tabular}{|c|c|c|c|c|c|c|}
\hline \multirow[b]{2}{*}{ Sn ion } & \multirow[b]{2}{*}{ Transition } & \multirow[b]{2}{*}{ Wavelength (nm) } & \multirow[b]{2}{*}{ Stark width (nm) } & \multicolumn{3}{|c|}{$d / w$} \\
\hline & & & & Expt. & Expt. (others) & Theory \\
\hline I & $5 p^{2}{ }^{3} P_{2}-5 p 6 s^{3} P_{1}$ & $317.60[23]$ & $0.093^{\mathrm{a}, \mathrm{b}}[52], 0.025^{\mathrm{a}}[53]$ & $0.32(5)$ & $+{ }^{\mathrm{c}}[70]$ & \\
\hline I & $5 p^{2}{ }^{1} D_{2}-5 p 6 s{ }^{1} P_{1}$ & $326.33[23]$ & $0.074^{\mathrm{a}, \mathrm{b}}[52], 0.036^{\mathrm{a}}[53], 0.080^{\mathrm{a}}[51]$ & $0.34(6)$ & & \\
\hline I & $5 p^{2}{ }^{1} D_{2}-5 p 6 s^{3} P_{2}$ & $333.16[23]$ & $0.045^{\mathrm{a}}[53]$ & $0.6(1)$ & & \\
\hline II & $5 s 5 p^{2}{ }^{2} D_{3 / 2}-5 s^{2} 4 f^{2} F_{5 / 2}$ & $328.41[23]$ & $\begin{array}{c}0.23^{\mathrm{a}, \mathrm{b}}[54], 0.022^{\mathrm{a}}[46], \\
0.038^{\mathrm{a}}[53], 0.16^{\mathrm{d}}[47], 0.13^{\mathrm{a}}[51]\end{array}$ & $0.26(2)$ & $0.22[46]$ & $0.38[47]$ \\
\hline II & $5 s 5 p^{2} D_{5 / 2}-5 s^{2} 4 f^{2} F_{5 / 2}$ & $335.29[23]$ & $\begin{array}{c}0.25^{\mathrm{a}, \mathrm{b}}[54], 0.047^{\mathrm{a}}[46] \\
0.044^{\mathrm{a}}[53], 0.24^{\mathrm{d}}[47]\end{array}$ & $0.24(2)$ & $0.20[46]$ & $0.21[47]$ \\
\hline III & $5 s 6 p^{1} P_{1}-5 s 6 d{ }^{1} D_{2}$ & $370.87[25]$ & $0.11^{\mathrm{b}, \mathrm{d}}[49]$ & $0.28(4)$ & & $0.38[49]$ \\
\hline III & $5 s 5 d^{1} D_{2}-4 f 5 s^{1} F_{3}$ & $390.79[25]$ & $0.088^{\mathrm{b}, \mathrm{d}}[49]$ & $0.21(6)$ & & $-0.11[49]$ \\
\hline IV & $5 d^{2} D_{3 / 2}-6 p^{2} P_{1 / 2}$ & $307.25[26]$ & $0.052^{\mathrm{d}}[58]$ & $0.05(2)$ & $0.09[56]$ & $-0.39[58]$ \\
\hline IV & $4 d^{9} 5 s^{2}{ }^{2} D_{5 / 2}-6 p^{2} P_{3 / 2}$ & $324.71[26]$ & $0.094^{\mathrm{d}}[58]$ & $0.19(5)$ & & $-0.49[58]$ \\
\hline IV & $6 s^{2} S_{1 / 2}-6 p^{2} P_{3 / 2}$ & $386.23[26]$ & $0.059^{\mathrm{b}, \mathrm{d}}[59], 0.11^{\mathrm{d}}[58]$ & $-0.12(1)$ & $-0.1[56]$ & $-0.38[58]$ \\
\hline IV & $6 s^{2} S_{1 / 2}-6 p^{2} P_{1 / 2}$ & $421.73[26]$ & $0.064^{\mathrm{b}, \mathrm{d}}[59], 0.087^{\mathrm{d}}[58]$ & $-0.17(2)$ & $-0.2[56]$ & $-0.48[58]$ \\
\hline
\end{tabular}

${ }^{\mathrm{a}}$ Experimental value.

${ }^{\text {b}}$ Parameter is used in Fig. 5.

${ }^{\mathrm{c} O n l y}$ the sign of the shift was established in Ref. [70].

${ }^{\mathrm{d}}$ Theoretical value. 


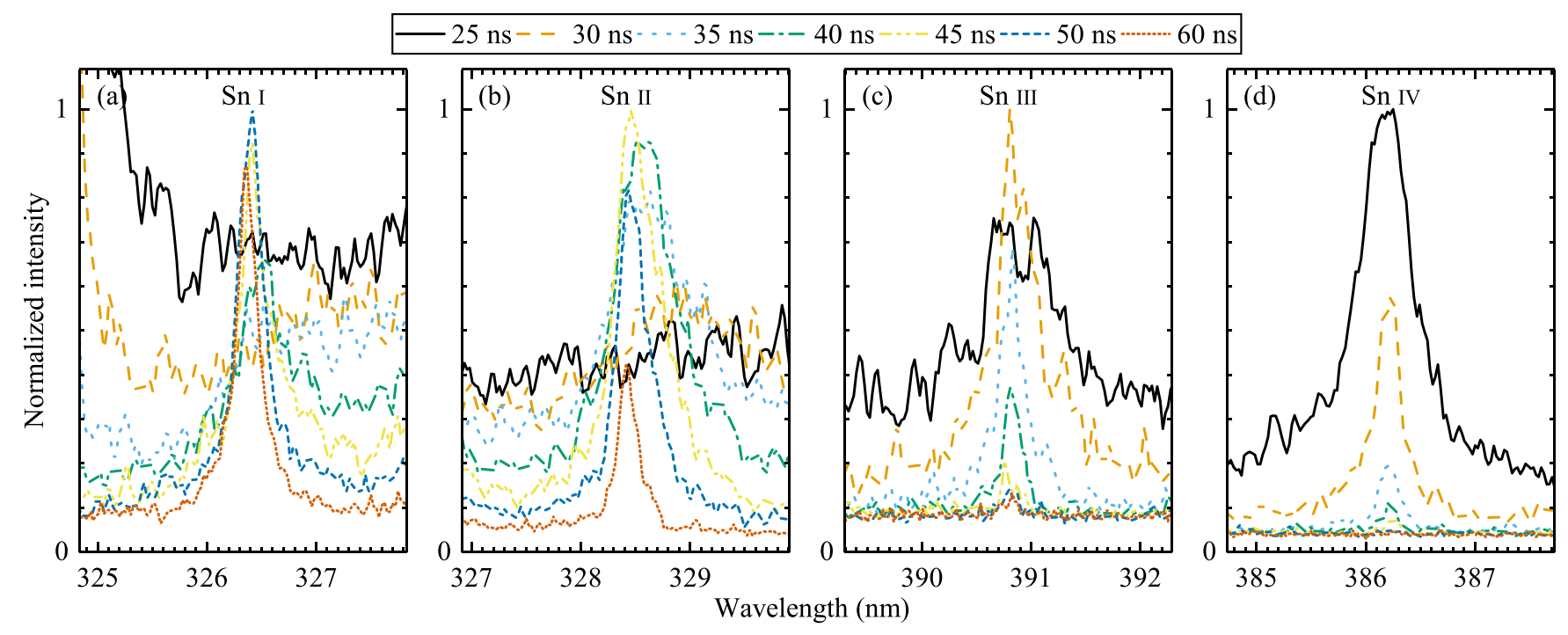

FIG. 2. Time-resolved spectra of selected lines belonging to Sn I-IV. Transitions are detailed in Table I. Lines are individually normalized to their respective maximum in time. Spectra shown are from plasma light captured by fiber core c, some $+250 \mu \mathrm{m}$ from the initial droplet position (see Fig. 1). Details of the individual transitions are given in Table I. Time is relative to the first moment of the 15-ns-long laser pulse hitting the droplet.

core [29]. In the following, we focus our studies mostly on spectra obtained from fibers $\mathbf{b}$, $\mathbf{c}$, and $\mathbf{d}$ that capture the outflow of plasma from the laser-matter interaction point; fiber core $\mathbf{b}$ probes the densest observable part of the plasma (see below).

\section{B. The Stark effect}

A widely used method for calculating Stark linewidths and shifts is the semiempirical (SE) approach of Griem [64], which is based on the original formulation by Baranger [65]. This generalized impact-broadening theory gives a description of the influence of perturbing electrons on lines subject to the linear Stark effect, neglecting the perturbing plasma ions for the current low densities and, in particular, low temperatures (see, e.g., Ref. [39], and references therein). Following, e.g., the work of de Andrés-García et al. [58] on the SE method, the half width at half maximum (in angular frequency units) linewidth $w_{\text {se }}$ of the Lorentz profile resulting from the Stark effect is given by

$$
\begin{aligned}
w_{\mathrm{se}} \approx & 8\left(\frac{\pi}{3}\right)^{3 / 2} \frac{\hbar}{m a_{0}} n_{e}\left(\frac{E_{\mathrm{H}}}{k T}\right)^{1 / 2} \\
& \times \sum_{i^{\prime}, f^{\prime}}\left[\left|\left\langle i^{\prime}|\boldsymbol{r}| i\right\rangle^{2} g_{\mathrm{se}}\left(\eta_{i}\right)+\right|\left\langle f^{\prime}|\boldsymbol{r}| f\right\rangle^{2} g_{\mathrm{se}}\left(\eta_{f}\right)\right] .
\end{aligned}
$$

The shift $d_{\text {se }}$ from the zero-electron-density position of the line is given, in angular frequency units, by

$$
\begin{aligned}
d_{\mathrm{se}} \approx & -8\left(\frac{\pi}{3}\right)^{3 / 2} \frac{\hbar}{m a_{0}} n_{e}\left(\frac{E_{\mathrm{H}}}{k T}\right)^{1 / 2} \\
& \times \sum_{i^{\prime}, f^{\prime}}\left[\left(\frac{\Delta E_{i^{\prime} i}}{\mid \Delta E_{i^{\prime} i}}\right) \mid\left\langle i^{\prime}|\boldsymbol{r}| i\right\rangle^{2} g_{\mathrm{sh}}\left(\eta_{i}\right)\right. \\
& \left.-\left(\frac{\Delta E_{f^{\prime} f}}{\mid \Delta E_{f^{\prime} f}}\right) \mid\left\langle f^{\prime}|\boldsymbol{r}| f\right\rangle^{2} g_{\mathrm{sh}}\left(\eta_{f}\right)\right],
\end{aligned}
$$

where $h$ is the Planck constant, $m$ is the electron mass, $a_{0}$ is the Bohr radius, $n_{\mathrm{e}}$ is the electron density, $T$ is the electron temperature, $k$ is the Boltzmann constant, $E_{\mathrm{H}}$ is the ionization energy of hydrogen, and $\Delta E_{i^{\prime} i}$ is the energy difference between a perturbing level $i^{\prime}$ and the perturbed level $i$. The indices $i$ and $f$ respectively indicate the upper (initial) and lower (final) levels belonging to the transitions; $\left\langle i^{\prime}|\boldsymbol{r}| i\right\rangle$ denotes the relevant dipole matrix element. The effective Gaunt factors $g_{\text {se }}\left(\eta_{i}\right)$ and $g_{\text {sh }}\left(\eta_{i}\right)$ are included as a quantummechanical correction factor $[66,67]$. These factors, of order unity, are slowly varying functions of $\eta_{i}=E /\left|\Delta E_{i^{\prime} i}\right|$ where $E$ is the mean energy of the perturbing electron, taken to be $E=(3 / 2) k T$. Theoretical calculations of the Stark shift are particularly challenging due to possible cancellation of terms in Eq. (2). These cancellations are absent in Eq. (1) and all terms add to the overall Stark width.

The dimensionless shift-to-width ratio $d_{\mathrm{se}} / w_{\mathrm{se}}$,

$$
\begin{aligned}
\frac{d_{\mathrm{se}}}{w_{\mathrm{se}}} \approx & \sum_{i^{\prime}, f^{\prime}}\left[\left|\left\langle i^{\prime}|\boldsymbol{r}| i\right\rangle^{2} g_{\mathrm{se}}\left(\eta_{i}\right)+\right|\left\langle f^{\prime}|\boldsymbol{r}| f\right\rangle^{2} g_{\mathrm{se}}\left(\eta_{f}\right)\right] / \\
& \sum_{i^{\prime}, f^{\prime}}\left[\left(\frac{\Delta E_{f^{\prime} f}}{\mid \Delta E_{f^{\prime} f}}\right) \mid\left\langle f^{\prime}|\boldsymbol{r}| f\right\rangle^{2} g_{\mathrm{sh}}\left(\eta_{f}\right)\right. \\
& \left.-\left(\frac{\Delta E_{i^{\prime} i}}{\mid \Delta E_{i^{\prime} i}}\right) \mid\left\langle i^{\prime}|\boldsymbol{r}| i\right\rangle^{2} g_{\mathrm{sh}}\left(\eta_{i}\right)\right]
\end{aligned}
$$

is independent of plasma parameters, aside from a possible weak dependence of the Gaunt factors on plasma temperature and uncertainties therein. The FWHM linewidth $w$, in wavelength units, is determined from $w=w_{\mathrm{se}} \lambda^{2} /(\pi c)$, with $\lambda$ the transition wavelength and $c$ the speed of light. In this work the $d / w$ ratio is used, based on the experimentally established FWHM width $w$ and shift $d$ in units of wavelength. This ratio serves as the basis for experimental tests of theory predictions. 
$25 \mathrm{~ns} \bigcirc 30 \mathrm{~ns} \Delta 35 \mathrm{~ns} \nabla 40 \mathrm{~ns}+45 \mathrm{~ns}<50 \mathrm{~ns} 60 \mathrm{~ns}$

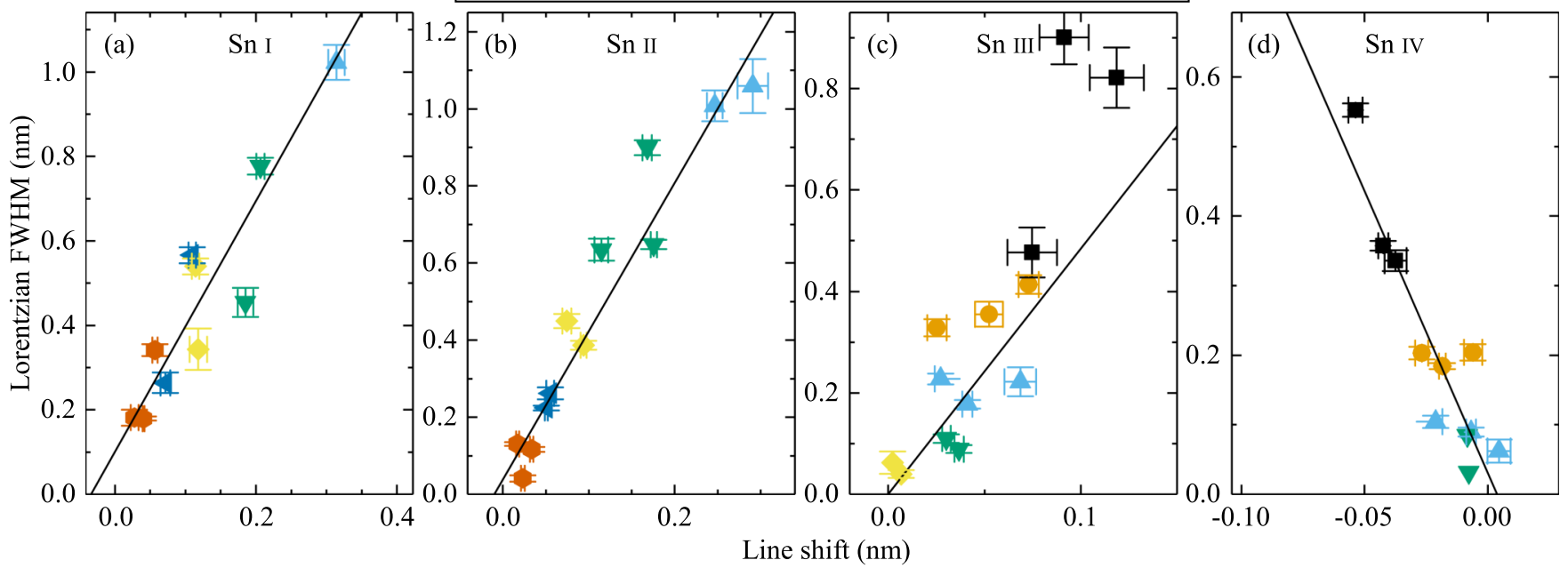

FIG. 3. Lorentzian widths against line shifts as obtained from fitting Voigt functions (with the respective Gaussian component inferred from nearby calibration lines) of the lines shown in Fig. 2. The shift is given with respect to literature values for the line position, listed in Table I. The results from the three fiber cores b, c, and $\mathbf{d}$ are shown (see Fig. 1). The error bars represent one standard deviation of the fit uncertainties. Solid straight lines represent linear fits to the data.

In Fig. 3, Lorentzian widths and shifts are displayed for the same transitions as shown in Fig. 2. These quantities are obtained from fitting Voigt functions to the spectral lines, which are convolutions of a Gaussian component representing the spectrometer's instrumental width, and a Lorentzian component representing the Stark effect. Doppler broadening is negligible at the $10^{-5}$ level. The fitting of more complex line profiles that could account for further broadening or other line-shape effects (see, e.g., Refs. [68,69]) are beyond the scope of this work. The respective Gaussian components are kept fixed at the value of the instrumental width obtained in the calibration procedure (nominally $\Delta \lambda=0.2 \mathrm{~nm}$ ). The Lorentzian components obtained from Voigt fits to the spectra obtained from three fiber cores $\mathbf{b}$, c, and $\mathbf{d}$ (see Fig. 1) are shown along with their respective shifts.

The data displayed in Fig. 3 demonstrate that width-toshift ratios, and thus their inverse the shift-to-width ratios $d / w$ are indeed constant. Solid straight lines represent linear fits to the concatenated data with free slope and intercepts. Self-absorption effects such as those observed at the densest, earliest times for a broadened Sn III line (see Fig. 2) may affect the interpretation of Stark line broadening. The potential impact of such opacity broadening may be assessed studying possible deviations from a straight line in the given shiftto-width ratios. Some deviations at the highest of densities may be tentatively identified but these do not significantly influence the fit. In all cases, the intercepts are consistent with the spectral line positions as listed in the available literature (see Table I). The slopes represent the shift-to-width ratios $d / w$. A complete list of the current results pertaining to $d / w$ ratios is given in Table I after careful selection of lines that are both sufficiently intense and sufficiently far separated from other lines. Values extracted from the available literature are normalized to an electron density of $10^{17} e^{-} \mathrm{cm}^{-3}$ and a temperature of $1 \mathrm{eV}$ (assuming a $T^{-1 / 2}$ dependency).
Our results are in good agreement with the available experimental work on Stark parameters. For the following comparison to model predictions, we note that the shift-towidth ratios are predicted to change negligibly (e.g., $<1 \%$ in the case of the here studied Sn IV lines [58]) over the here relevant temperature range.

\section{Sn I}

Stark broadening of Sn I lines has been investigated in several experimental $[41,51-54]$ and theoretical $[40,50]$ studies. We observe three Sn I lines at wavelengths near $320 \mathrm{~nm}$, all $5 p^{2}-5 p 6 s$ transitions. Experimental Stark widths for these lines are presented in Refs. [51-53]. The values reported by Ref. [53] are a factor of 2-4 lower than presented by the other authors [51,52].

Experimental studies on the sign of the Stark shift of Sn Ilines are presented in Ref. [70], as part of a systematic study on the neutral atoms in the carbon group. No quantitative data on the magnitude of the shift are available in the literature. Our findings agree with the findings in Ref. [70] that the $5 p^{2}{ }^{3} P_{2}-5 p 6 s^{3} P_{1}$ transition shifts towards longer wavelength; as do the other $5 p^{2}-5 p 6 s$ transitions (see Table I).

\section{Sn II}

Both experimental [46,51,53,54] and theoretical [47] Stark effect data is available for the here studied lines of Sn II. Theory predictions are in good agreement with our findings for the $5 s 5 p^{2} D_{5 / 2}-5 s^{2} 4 f^{2} F_{5 / 2}$ transition, whereas a discrepancy can be identified for the other line of the multiplet, the $5 s 5 p^{2} D_{3 / 2}-5 s^{2} 4 f^{2} F_{5 / 2}$. In comparing our findings with previous experiments (see Table I) excellent agreement is found for the $d / w$ ratio. 


\section{Sn III}

Several experimental studies on the Stark broadening of Sn III lines were previously conducted $[29,48,55]$, albeit in a wavelength regime generally below $300 \mathrm{~nm}$, outside the range of our studies. SE calculations for a more complete set of transitions are presented in Ref. [49]. These calculations are in good agreement with previously available experimental data. The two Sn III lines studied in this work both shift towards longer wavelength. The SE calculations, however, predict a negative shift for the $5 s 5 d^{1} D_{2}-4 f 5 s^{1} F_{3}$ transition and a positive shift for the $5 s 6 p^{1} P_{1}-5 s 6 d{ }^{1} D_{2}$ transition (see Table I).

\section{Sn IV}

In the case of Sn IV, various experimental results for a selection of lines are available [55-57]. Our experimental findings are in excellent agreement with these previous findings, where available. Theoretical calculations, using a modified-SE method, of a selection of lines can be found in Ref. [59]. SE calculations for a larger set of transitions are presented in Ref. [58] and these are used for the comparison of $d / w$ ratios in Table I. Our experimental findings are in disagreement with the calculations of the shift-over-width values. In two out of the four cases this disagreement extends to the sign of the Stark shift.

\section{DIAGNOSTICS OF THE AFTERGLOW OF LASER-PRODUCED PLASMA}

The Nd:YAG-laser-produced tin plasma relevant for the production of extreme ultraviolet light is hot $(\sim 30 \mathrm{eV})$ and dense $\left(\sim 10^{21} e^{-} \mathrm{cm}^{-3}\right)$ (see, e.g., Refs. [62,72,73]). At the laser intensity of $1.7 \times 10^{11} \mathrm{~W} / \mathrm{cm}^{2}$, used in our experiments, given the density set by the laser wavelength [1], the temperature of the plasma produces a charge-state distribution containing mostly Sn XII-Sn XV ions [73] that is optimal for emitting EUV light at $13.5 \mathrm{~nm}[62,72]$. After the laser pulse ends, the plasma quickly cools down while continuing its free, quasispherical expansion [74]. Optical emission is readily observed in this afterglow phase. Stark widths obtained from lines in this optical domain can assist in diagnosing plasma afterglow regarding its electron density and scaling thereof with time and position.

For the purpose of determining the electron density, the plasma electron temperature $T$ should be known only to a reasonable accuracy, given the rather weak $\sqrt{T}$ dependence of $w$. The Saha-Boltzmann equation, following the procedure outlined in Ref. [75], can provide the required information based on a fit of observed emission line strengths, as a function of excitation and ionization energies. It assumes local thermodynamic equilibrium (LTE) conditions. Regarding the assumption of LTE, first, we note that the McWhirther criterion [76] for LTE is well met over the here relevant plasma densities and temperatures. Second, we performed FLYCHK [71] calculations of the average charge state $\bar{z}$ over a range of electron temperatures and densities, both under LTE and non-LTE conditions and found no significant differences over the here relevant densities and temperatures.

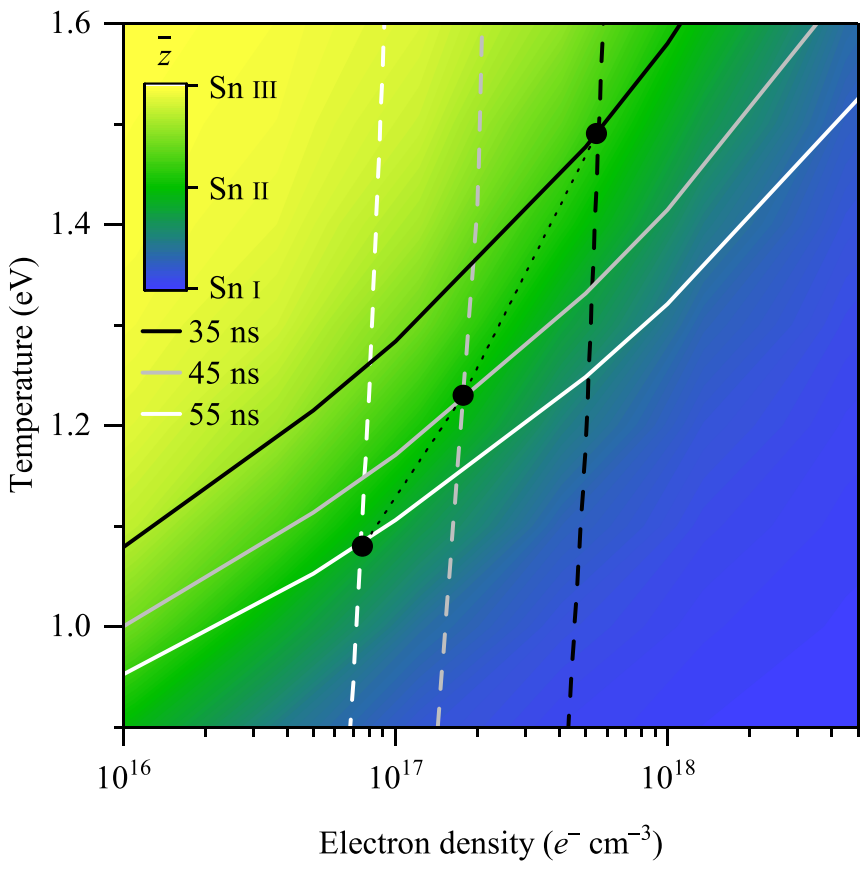

FIG. 4. Color map of the average charge state $\bar{z}$ as a function of electron density and temperature from FLYCHK calculations [71] in LTE. The solid (near diagonal) lines indicate the solutions allowed by a Saha-Boltzmann fit to Sn I and Sn II lines, performed for each time step for fiber core b (see Fig. 1). The dashed (near vertical) lines indicate allowed solutions for the electron densities (as a weak function of $T$ ) as inferred from the width of the Sn II $5 s 5 p^{2}{ }^{2} D_{3 / 2}-5 s^{2} 4 f^{2} F_{5 / 2}$ transition at the 328-nm line using the experimental input from Ref. [54] (see main text). The intersections of solid and dashed lines identify unique solutions for electron density and temperature. Time is relative to the first moment of the 15-ns-long laser pulse hitting the droplet.

Figure 4 presents our FLYCHK calculations, under LTE conditions, of $\bar{z}$ over the relevant range of electron temperatures and densities. Overlaid on the interpolated FLYCHK output are the allowed solutions in (density, temperature) space resulting from fits of the Saha-Boltzmann equation to emission line strengths of Sn I and Sn II for selected time steps as observed in fiber core b (see Fig. 1). These allowed solutions are presented by solid lines in Fig. 4. These solutions are in broad agreement with estimates (not shown) of the average charge state based just on the ratios of emission line strengths of the various charge states. Uncertainties of up to several tens of percent in our temperature estimation should be taken into account. The dashed, near-vertical lines indicate the allowed solutions in (density, temperature) space resulting from the observed width of the Sn II 328-nm line from fiber core $\mathbf{b}$, taking as input the experimentally determined width parameter from Ref. [54]. The intersections of solid and dashed lines, indicated by black dots, identify the unique solutions for electron density and temperature in a time- (and, in principle, space-) resolved manner. A clear reduction of plasma temperature, and a much faster reduction of electron density, is thus observed in the time window from 35 to $55 \mathrm{~ns}$. Given the observed modest change in plasma temperature, and 


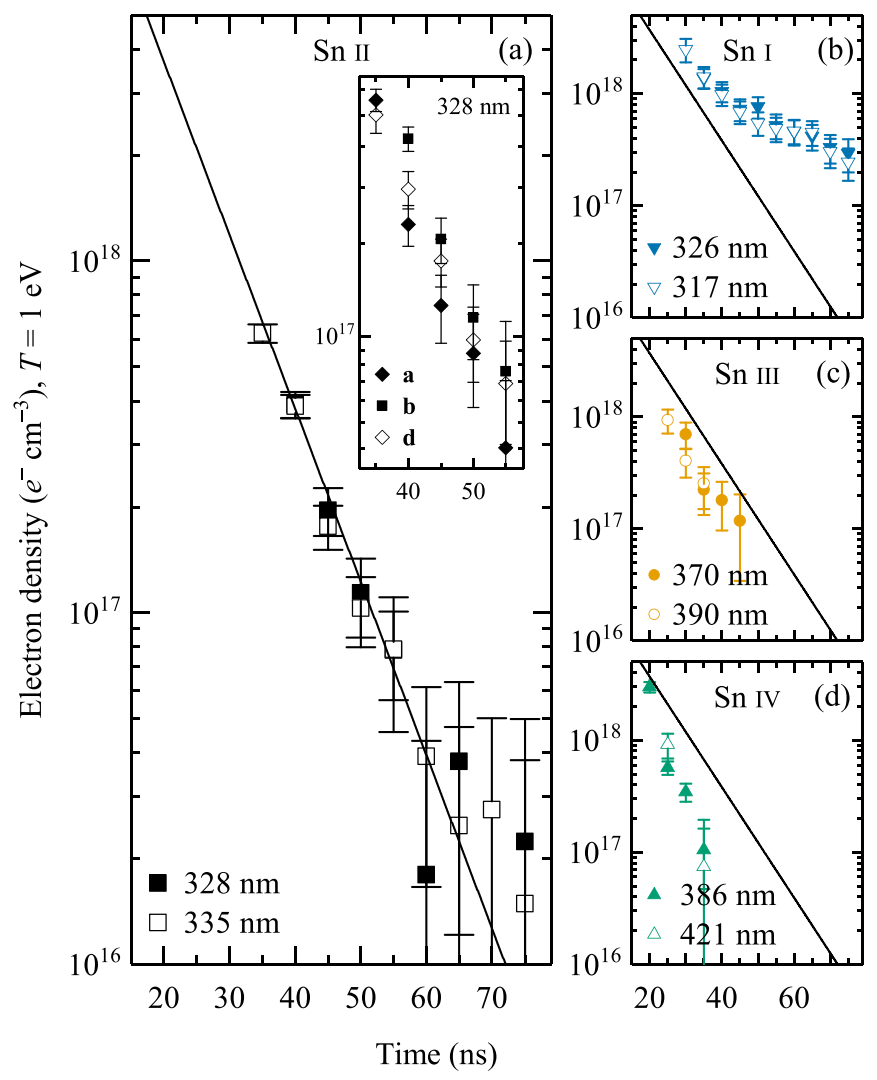

FIG. 5. Plasma electron density determined from measurements taken at fiber core b (see Fig. 1) as a function of time (relative to the first moment of the 15-ns-long laser pulse hitting the droplet). The densities are calculated using the experimental Stark width in the case of Sn II [54], and theoretical widths from Sn I [52], Sn III [51], and Sn IV [59] taken at their $T=1 \mathrm{eV}$ values; details are given in Table I. Uncertainty estimates combine uncertainties on the input parameters (a $20 \%$ error margin is assumed where no value was provided), with statistical ones and a constant $0.05 \mathrm{~nm}$ uncertainty that captures systematic deviations seen, e.g., in Fig. 3. The solid black line results from an exponential fit to the $328-\mathrm{nm}$ data of Sn II. The inset in panel (a) shows 328-nm data also from fiber cores a and d (see Fig. 1). Panels (b), (c), and (d) show the densities as derived from lines of Sn I, Sn III, and Sn IV, respectively, from fiber core $\mathbf{b}$. The black lines are identical to the one shown in panel (a).

weak dependence of the Stark width on temperatures of $\sim 1 \mathrm{eV}$, its time evolution is not explicitly considered in the following.

Figure 5(a) shows the evolution of plasma electron density with time as obtained from fiber core b (see Fig. 1) from our studies of the two lines (see Table I) from Sn II, taking a constant $T=1 \mathrm{eV}$ and using the experimentally determined width parameters from Miller et al. [54] who provide values for both here observed Sn II lines. These values agree with those from other experiments [51] and calculations [47] within roughly a factor of 2 that is typical for these complicated experiments and calculations. Experimental values from Ref. [53] are somewhat further removed from the other works. Using the width parameters of Miller et al., both Sn II lines show consistent results regarding electron densities [cf.
Fig. 5(a)]. An exponential decay of electron density with time is observed, with approximately a decade drop in density every 20 ns. The black solid line shows the result of an exponential fit to the data of the 328-nm line. No significant change in density is observed when shifting the location of our observations $+250 \mu \mathrm{m}$ in the direction of the laser origin (fiber core d), or $-125 \mu \mathrm{m}$ away from it towards the original droplet location (fiber core a), as shown in the inset, indicating a spatially rather flat plasma density profile with the highest density observed through fiber core $\mathbf{b}$.

Also shown are the plasma densities as derived from linewidths in Sn I, Sn III, and Sn IV ions in panels (b), (c), and (d), respectively. The required theoretical input values for the width parameters at $T=1 \mathrm{eV}$ are obtained from Ref. [52] for Sn I, Ref. [51] for Sn III, and Ref. [59] for Sn IV. Note that the various literature values for the broadening parameters may differ again up to a factor of 5 (see Table I). The black solid lines are identical to the one shown in panel (a). Given the uncertainties on the required input values, the agreement between the results obtained from the various charge states at early times is reasonable.

While being aware of the sizable uncertainties involved in the Stark broadening parameters, a charge-state-dependent trend can tentatively be identified in the data. This trend would indicate that the lower charge states appear to probe a higher plasma density, while the higher charge states probe a lower density, all compared to Sn II. These differences increase with time. The trend may originate from our line-of-sight integration of emission intensity. Alternatively, the trend may point towards here unexplored contributions in the Stark parameters that serve as input.

\section{CONCLUSION}

The Stark effect was observed in optical lines in the emission from laser-produced tin plasma in the afterglow phase 20-40 ns after the end of the 15-ns-long temporally boxshaped laser pulse that illuminates the tin droplets. Time- and space-resolved spectroscopy of Sn I-IV ions was performed of 11 lines from Sn I-IV ions, in an 315-425-nm wavelength range. Stark shift-to-width ratios, which are here largely independent of temperature and density, served as the basis for experimental tests of atomic physics theory predictions. These predictions are found to be in poor agreement with the experimental data and cases are found where theory and experiment disagree on the sign of the Stark line shift. Previous experimental input for the Stark width parameter was used, in tandem with Saha-Boltzmann fits of Sn I and Sn II lines, to obtain the temperature and density evolution of the plasma with time. A strong, exponential reduction of density from $\sim 10^{18}-10^{17} e^{-} \mathrm{cm}^{-3}$ in a 20-ns time window, was established using the Sn II lines. A clear cool-down from a $\sim 2$ to $1 \mathrm{eV}$ temperature is observed in this same time window, having started at $\sim 30 \mathrm{eV}$, and $\sim 10^{21} e^{-} \mathrm{cm}^{-3}$, when emitting EUV light during the application of the 15-ns-long laser pulse. No significant difference in density was observed from our studies of Sn II when translating the region of observation $250 \mu \mathrm{m}$ in the direction of the laser beam origin, indicating, locally, a spatially rather flat plasma density profile. Stark widths of 
the other charge states Sn I, III, and IV gave similar density values at early times but a tentative trend was established where, over time, lower charge states probe higher plasma densities.

Our work is relevant for understanding the interaction of the expanding plasma with the local environment in an EUV nanolithography machine. Future studies could include higher charge states with observable transitions in the deep ultraviolet range to be able to probe the dense part of the plasma most relevant for the production of EUV light.

\section{ACKNOWLEDGMENTS}

This work was carried out at the Advanced Research Center for Nanolithography, a public-private partnership between the University of Amsterdam, the Vrije Universiteit Amsterdam, the Netherlands Organization for Scientific Research (NWO), and the semiconductor equipment manufacturer ASML. This project received funding from the European Research Council (ERC) Starting Grant No. 802648 and is part of the VIDI research program with Project No. 15697, which is financed by NWO.
[1] O. O. Versolato, Plasma Sources Sci. Technol. 28, 083001 (2019).

[2] J. Benschop, V. Banine, S. Lok, and E. Loopstra, J. Vac. Sci. Technol. B 26, 2204 (2008).

[3] V. Y. Banine, K. N. Koshelev, and G. H. P. M. Swinkels, J. Phys. D 44, 253001 (2011).

[4] G. O’Sullivan, B. Li, R. D'Arcy, P. Dunne, P. Hayden, D. Kilbane, T. McCormack, H. Ohashi, F. O’Reilly, P. Sheridan, E. Sokell, C. Suzuki, and T. Higashiguchi, J. Phys. B 48, 144025 (2015).

[5] F. Torretti, F. Liu, M. Bayraktar, J. Scheers, Z. Bouza, W. Ubachs, R. Hoekstra, and O. Versolato, J. Phys. D 53, 055204 (2019).

[6] A. Bayerle, M. J. Deuzeman, S. van der Heijden, D. Kurilovich, T. de Faria Pinto, A. Stodolna, S. Witte, K. S. E. Eikema, W. Ubachs, R. Hoekstra, and O. O. Versolato, Plasma Sources Sci. Technol. 27, 045001 (2018).

[7] J. W. Coenen, G. De Temmerman, G. Federici, V. Philipps, G. Sergienko, G. Strohmayer, A. Terra, B. Unterberg, T. Wegener, and D. C. M. van den Bekerom, Phys. Scr. 2014, 014037 (2014).

[8] G. G. van Eden, T. W. Morgan, D. U. B. Aussems, M. A. van den Berg, K. Bystrov, and M. C. M. van de Sanden, Phys. Rev. Lett. 116, 135002 (2016).

[9] G. G. Eden, V. Kvon, M. C. M. van de Sanden, and T. W. Morgan, Nat. Commun. 8, 192 (2017).

[10] T. W. Morgan, P. Rindt, G. G. van Eden, V. Kvon, M. A. Jaworksi, and N. J. L. Cardozo, Plasma Phys. Control. Fusion 60, 014025 (2017).

[11] S. J. Adelman, W. P. Bidelman, and D. M. Pyper, Astrophys. J. Suppl. Ser. 40, 371 (1979).

[12] L. M. Hobbs, D. E. Welty, D. C. Morton, L. Spitzer, and D. G. York, Astrophys. J. 411, 750 (1993).

[13] B. D. Savage and K. R. Sembach, Annu. Rev. Astron. Astrophys. 34, 279 (1996).

[14] U. J. Sofia, D. M. Meyer, and J. A. Cardelli, Astrophys. J. Lett. 522, L137 (1999).

[15] C. R. Proffitt, C. J. Sansonetti, and J. Reader, Astrophys. J. 557, 320 (2001).

[16] P. Chayer, S. Vennes, J. Dupuis, and J. W. Kruk, Astrophys. J. Lett. 630, L169 (2005).

[17] S. J. O’Toole, Astron. Astrophys. 423, L25 (2004).

[18] S. Vennes, P. Chayer, and J. Dupuis, Astrophys. J. 622, L121 (2005).

[19] S. J. O'Toole and U. Heber, Astron. Astrophys. 452, 579 (2006).
[20] K. Werner, T. Rauch, E. Ringat, and J. W. Kruk, Astrophys. J. 753, L7 (2012).

[21] S. Biswas, A. Das, A. Bhowmik, and S. Majumder, Mon. Not. R. Astron. Soc. 477, 5605 (2018).

[22] M. Dorsch, M. Latour, and U. Heber, Astron. Astrophys. 630, A130 (2019).

[23] A. Kramida, Yu. Ralchenko, J. Reader, and NIST ASD Team, NIST Atomic Spectra Database (ver. 5.5.6), National Institute of Standards and Technology, Gaithersburg, MD (2018), https://physics.nist.gov/asd.

[24] K. Haris, A. Kramida, and A. Tauheed, Phys. Scr. 89, 115403 (2014).

[25] K. Haris and A. Tauheed, Phys. Scr. 85, 055301 (2012).

[26] J. Scheers, A. Ryabtsev, A. Borschevsky, J. C. Berengut, K. Haris, R. Schupp, D. Kurilovich, F. Torretti, A. Bayerle, E. Eliav, W. Ubachs, O. O. Versolato, and R. Hoekstra, Phys. Rev. A 98, 062503 (2018).

[27] R. W. Coons, S. S. Harilal, D. Campos, and A. Hassanein, J. Appl. Phys. 108, 063306 (2010).

[28] H. Lan, H. Lei, D. Zuo, X. Wang, and G. Zheng, Vacuum 135, 86 (2017).

[29] E. R. Kieft, J. J. A. M. van der Mullen, G. M. W. Kroesen, V. Banine, and K. N. Koshelev, Phys. Rev. E 70, 066402 (2004).

[30] S. Namba, S. Fujioka, H. Sakaguchi, H. Nishimura, Y. Yasuda, K. Nagai, N. Miyanaga, Y. Izawa, K. Mima, K. Sato, and K. Takiyama, J. Appl. Phys. 104, 013305 (2008).

[31] J. Iqbal, R. Ahmed, M. Rafique, M. A. ul Haq, and M. A. Baig, Laser Phys. 26, 076001 (2016).

[32] S. S. Harilal, B. O'Shay, M. S. Tillack, and M. V. Mathew, J. Appl. Phys. 98, 013306 (2005).

[33] A. Roy, S. S. Harilal, S. M. Hassan, A. Endo, T. Mocek, and A. Hassanein, Laser Part. Beams 33, 175 (2015).

[34] H. R. Griem, Spectral Line Broadening by Plasmas (Academic, New York, 1974).

[35] A. Hauer, K. G. Whitney, P. C. Kepple, and J. Davis, Phys. Rev. A 28, 963 (1983).

[36] A. Lesage, New Astron. Rev. 52, 471 (2009).

[37] N. Konjević, A. Lesage, J. R. Fuhr, and W. L. Wiese, J. Phys. Chem. Ref. Data 31, 819 (2002).

[38] A. Kramida and J. Fuhr, Atomic Line Broadening Bibliographic Database (version 3.0), National Institute of Standards and Technology, Gaithersburg, MD, https://physics.nist.gov/ linebrbib.

[39] S. Sahal-Bréchot, M. S. Dimitrijević, and N. Moreau, STARKB database, Observatory of Paris, LERMA and Astronomical Observatory of Belgrade (2020), http://stark-b.obspm.fr. 
[40] M. S. Dimitrijević and N. Konjević, J. Quant. Spectrosc. Radiat. Transf. 30, 45 (1983).

[41] S. Djeniže, A. Srećković, J. Labat, R. Konjević, and M. Brnović, Z. Phys. D 24, 1 (1992).

[42] B. Blagojević and N. Konjević, J. Quant. Spectrosc. Radiat. Transf. 198, 9 (2017).

[43] J. Purić, M. Ćuk, and I. S. Lakićević, Phys. Rev. A 32, 1106 (1985).

[44] J. D. Hey and P. Breger, J. Quant. Spectrosc. Radiat. Transf. 23, 311 (1980).

[45] I. S. Lakićević and J. Purić, J. Phys. B 16, 1525 (1983).

[46] S. Djeniže, A. Srećković, and J. Labat, Z. Phys. D 17, 85 (1990).

[47] C. Colón, A. Alonso-Medina, C. Rivero, and F. Fernández, Phys. Scr. 73, 410 (2006).

[48] Z. Simić, M. S. Dimitrijević, A. Kovačević, and M. Dačić, Publ. Astron. Obs. Belgrade 84, 487 (2008).

[49] A. Alonso-Medina and C. Colón, Mon. Not. R. Astron. Soc. 414, 713 (2011).

[50] H. M. Johns, D. P. Kilcrease, J. Colgan, E. J. Judge, J. E. Barefield II, R. C. Wiens, and S. M. Clegg, J. Phys. B 48, 224009 (2014).

[51] A. Alonso-Medina and C. Colón, Astrophys. J. 672, 1286 (2008).

[52] B. Martinez and F. Blanco, J. Phys. B 32, 241 (1999).

[53] S. Djeniže, A. Srećković, and Z. Nikolić, J. Phys. B 39, 3037 (2006).

[54] M. H. Miller, R. A. Roig, and R. D. Bengtson, Phys. Rev. A 20, 499 (1979).

[55] S. Djeniže, Spectrochim. Acta B 62, 403 (2007).

[56] M. A. Mazing and N. A. Vrublevskaya, Opt. Spectrosc. (USSR) (English Transl.) 16, 6 (1964).

[57] M. Burger, M. Skočić, M. Gavrilov, S. Bukvić, and S. Djeniže, Publ. Atron. Obs. Belgrade 91, 53 (2012).

[58] I. de Andrés-García, A. Alonso-Medina, and C. Colón, Mon. Not. R. Astron. Soc. 455, 1145 (2016).

[59] M. S. Dimitrijević, Z. Simić, R. Stamm, J. Rosato, N. Milovanović, and C. Yubero, Atoms 6, 10 (2018).
[60] H. R. Griem, Principles of Plasma Spectroscopy (Cambridge University Press, Cambridge, UK, 2005).

[61] R. A. Meijer, A. S. Stodolna, K. S. E. Eikema, and S. Witte, Opt. Lett. 42, 2758 (2017).

[62] R. Schupp, F. Torretti, R. A. Meijer, M. Bayraktar, J. Scheers, D. Kurilovich, A. Bayerle, K. S. E. Eikema, S. Witte, W. Ubachs, R. Hoekstra, and O. O. Versolato, Phys. Rev. Appl. 12, 014010 (2019).

[63] D. Kurilovich, A. L. Klein, F. Torretti, A. Lassise, R. Hoekstra, W. Ubachs, H. Gelderblom, and O. O. Versolato, Phys. Rev. Appl. 6, 014018 (2016).

[64] H. R. Griem, Phys. Rev. 165, 258 (1968).

[65] M. Baranger, Phys. Rev. 112, 855 (1958).

[66] M. Seaton, Atomic and Molecular Processes, edited by D. Bates, Vol. 374 (Academic, New York, 1962).

[67] H. van Regemorter, Astrophys. J. 136, 906 (1962).

[68] V. Milosavljević and G. Poparić, Phys. Rev. E 63, 036404 (2001).

[69] N. Konjević, Phys. Rep. 316, 339 (1999).

[70] E. V. Kondratyeva, Opt. Spectrosc. (USSR) (English Transl.) 28, 449 (1970).

[71] H.-K. Chung, M. H. Chen, W. L. Morgan, Y. Ralchenko, and R. W. Lee, High Energy Density Phys. 1, 3 (2005).

[72] R. Schupp, F. Torretti, R. A. Meijer, M. Bayraktar, J. Sheil, J. Scheers, D. Kurilovich, A. Bayerle, A. A. Schafgans, M. Purvis, K. S. E. Eikema, S. Witte, W. Ubachs, R. Hoekstra, and O. O. Versolato, Appl. Phys. Lett. 115, 124101 (2019).

[73] F. Torretti, J. Sheil, R. Schupp, M. M. Basko, M. Bayraktar, R. A. Meijer, S. Witte, W. Ubachs, R. Hoekstra, O. O. Versolato, A. J. Neukirch, and J. Colgan, Nat. Commun. 11, 2334 (2020).

[74] M. M. Basko, V. G. Novikov, and A. S. Grushin, Phys. Plasmas 22, 053111 (2015).

[75] J. A. Aguilera and C. Aragón, Spectrochim. Acta B 59, 1861 (2004).

[76] T. Fujimoto and R. W. P. McWhirter, Phys. Rev. A 42, 6588 (1990). 